Vol.02/ No. 02

Pages: 102-111

https://www.irojournals.com/iroeea

DOI: https://doi.org/10.36548/jeea.2020.2.006

\title{
Flawless Attuning for Parameters of Power System Modulator Applying Grey Wolf Optimization
}

\author{
Dr. A. Dinesh Kumar, \\ Computer Science and Engineering, \\ Koneru Lakshmaiah Education Foundation, \\ Andra Pradesh, India. \\ Email: adinesh@kluniversity.in
}

\begin{abstract}
Flawless attuning for the parameters of the power system regulator/ stabilizer / modulator is presented in the paper. The attuning of parameters in the power system modulator (PSM) becomes a prerequisite to have a stable and reliable performance across a broad scope of operating as well as system conditions. The parameter choices in the PSM for concurrently stabilizing the oscillation in the system is often transformed into an unpretentious optimization issue. This optimization issue in the proposed method is solved by foraging behaviors of the grey wolves. The proficiency of the mechanism devised in the paper is evaluated with two test cases of a Multi-device system encompassed with two machines and four buses. The results observed from the test cases prove that the attuning of the parameters using the foraging behavior of the grey wolves is a smart another to the traditionalFGM (fixed gain modulators/stabilizers). The experimental outcomes for the proposed method shows that the parameter attuning devised by the GWO affords to deliver a most compatible, reliable and a stabilized overall system performance compared to the traditional techniques.
\end{abstract}

Keywords: Attuning, PSM, PSM-Parameters, Grey Wolf Optimization, Stable Performance

\section{Introduction}

The robust functioning of the power system that are interconnected demands for a perfect management of the real and the reactive power. The changes in the actual power disturbs the frequency of the system and the variations in the terminal voltages directly affects the reactive power that is highly sensitive to the changes in the power systems terminal voltages. So a load frequency stabilizer and the automated voltage stabilizer is used to regulate the real power and reactive power respectively and the terminal voltage as well.

The application of the stabilizers for the power system could regulate both the real and the reactive power. These stabilizer/modulators/ regulators are incorporated into a single generator that is utilized in providing the additional regulation signals, for the excitation operation of the generator.

ISSN: 2582-3051 
Journal of Electrical Engineering and Automation (EEA) (2020)

Vol.02/ No. 02

Pages: 102-111

https://www.irojournals.com/iroeea

DOI: https://doi.org/10.36548/jeea.2020.2.006

The PSM is device that measures enhancements in the stability of the system when included to automated voltage stabilizer of the generator. When compared to the rebuilding of the system or improving it the PSM affords to offer an overpowering, grander, cost -effective operation. This is caused by the abundant system line up integrating the analog the digital and the input type frameworks $(\Delta p, \Delta w, \Delta f)$. The PSM is mainly utilized to regulate the excitation values and minimize the swift fluctuations in the power. The table.1 below shows the description of the torque vector achieved for the constant excitation, the automated voltage stabilizer and the automated voltage stabilizer integrated with the PSM.

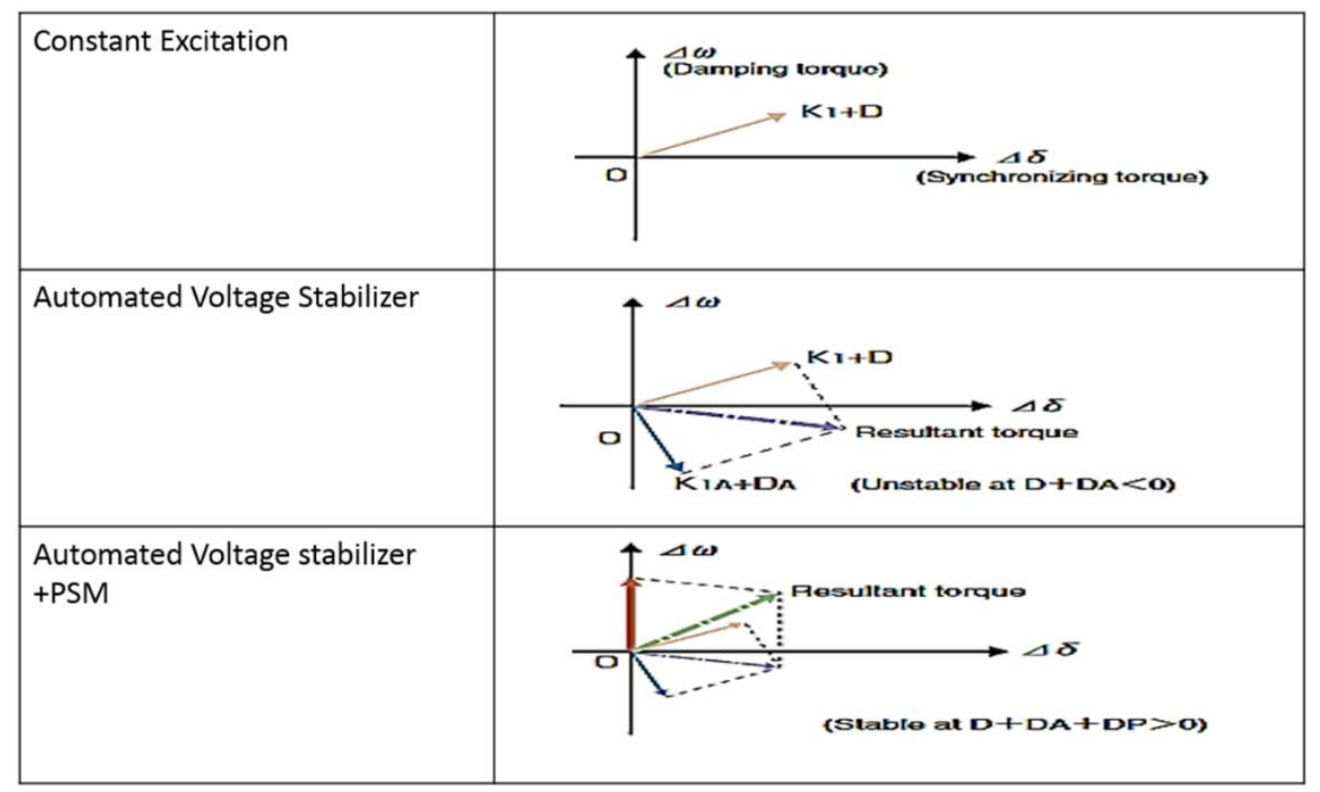

Table.1 Torque Characteristics [1]

Though attuning the parameters of the power system modulator (PSM) delivers a stable and reliable performance across a broad scope of operating as well as system conditions. The parameter choices in the PSM for concurrently stabilizing the oscillation in the system is often transformed into an unpretentious optimization issue. This optimization issue in the proposed method is solved by foraging behaviors of the grey wolves

The remaining paper is organized with the related works in part two, The foraging behavior of grey wolves in attuning the power system parameters in part three, the evaluation using the test cases in part 4 and the conclusion in part 5. 
Journal of Electrical Engineering and Automation (EEA) (2020)

Vol.02/ No. 02

Pages: 102-111

https://www.irojournals.com/iroeea

DOI: https://doi.org/10.36548/jeea.2020.2.006

\section{Related Works}

The parameters of the PSM are capable of delivering a stable operation for the multiple plants by the attuning done employing the intelligent algorithms like the "particle swarm optimization" as proposed by El-Zonkoly, et al [2] "harmony search algorithm" put forth by Naresh, G., et al [3] "grass hopper optimization" put forward by Ali, E. S., et al [3] , "farm land feritility optimization" performed by Sabo et al [5] using "salp swarm algorithm" that is in Ekinci et al [6] or by employing the "whale optimization algorithm" that was performed by Dasu, Butti et al [7] the proposed method in the paper also scopes in attuning the parameters of the PSM but by applying the foraging behavior of the grey wolves, the following paper in the related works section elaborates the need for the stabilization the authors

Gurrala, et al [8] elaborates the necessary design modification required in the power system that are inter linked and suggests the incorporation of the power system regulator to attain a stable operation. Lefebvre, $S$ et al [9] the necessity and the methodology of tuning for the stabilizers utilized in a multi machine system. Priyamvada et al [10] has performed the "Robust Control based Energy Storage Stabilizers for Power Systems with Large Scale Renewables." Nikolaev et al [11] elaborates the. "Study of Different Types of Power Systems Stabilizers used in Synchronous Generators Automatic Excitation Control Systems." Chow, et al [12] presents the insights on the necessity of the power system stabilizers to improve the performance and minimize the fluctuations in the output. Raj, Jennifer S et al [13] discusses the "Comprehensive Survey on the Computational Intelligence Techniques and Its Applications." Manikandan, R., et al [14] has devised a "Controller based performance measures of speed control of electrical motor for industrial applications." the " review of latest modifications and uses of the grey wolf optimization was presented by Faris, Hossam, et al [15]

\section{Parameter Attuning For PSM Applying Grey Wolf Optimizer}

The section provides the general phases involved in the foraging behavior of the grey wolves and then describes the proposed algorithm for attuning the parameters of the PSM.

Grey Wolf Optimizer: the algorithm is completely based on the leadership chain of command, and the hunting strategy followed by the grey wolves. The pack of wolves that come under the clan of Canidae are considered as intruders so the wolves maintain a command chain as alpha (A), beta (B), Delta (D) and omega (O). The A-group of wolves are the top most in the command chain and are considered to be leaders. The complete decision making such as the time to hunt sleep and wake is managed by the A-group, this decision from the $\mathrm{A}$ is communed to the other groups $\mathrm{B}, \mathrm{D}$ and $\mathrm{O}$. B are the subsidiary to A. the third stage wolves come under $\mathrm{O}$ group that helps in retaining the dominant structure of the hierarchy. The $\mathrm{D}$ are the 
Journal of Electrical Engineering and Automation (EEA) (2020)

Vol.02/ No. 02

Pages: 102-111

https://www.irojournals.com/iroeea

DOI: https://doi.org/10.36548/jeea.2020.2.006

left over pack of wolves that neither com under the A,B,O, the D type follows the A and B and controls the $\mathrm{O}$, the wolf pack is categorized into five types as Scouts (Sc), Sentinels (Se), Elders(E), then as hunters $(\mathrm{H})$ and care takers $(\mathrm{CT})$ for the purpose of guarding, ensuring safety, teaching on experiences, hunting and taking care respectively

There are four phases in hunting for a grey wolf, the first stage begins with simple search, and the wolves separate themselves in different direction and searches for food and gathers back once the location of the food is sorted. Once the food is found the food is encircled this is the next phase in the hunting. The equation 1 depicts the mathematical formula for the encircling.

$$
\overrightarrow{\text { Encircling }}=\mid \overrightarrow{O B} \text { c. } \vec{P}_{p}(j)-\vec{P}(j) \mid
$$

Where $\mathrm{P}$ is the prey and the $\mathrm{OB}$ is the obstacle. $\vec{P}_{p}$ is the position of prey, the ' $\mathrm{M}$ ' is a coefficient that retains the distance between the prey and the pack. Once encircled the next phase that is the hunting begins as instructed by the A,B and $\mathrm{O}$, the fittest optimal are delivered by the $\mathrm{A}$ the hunting behavior is mathematically depicted as shown in equation 2

$$
\begin{aligned}
& \overrightarrow{\text { Enclrcling }}_{A}=\mid \overrightarrow{O B 1} \text { c. } \vec{P}_{A}(j)-\vec{P}(j) \mid \\
& \overrightarrow{\text { Encırcling }}_{B}=\mid \overrightarrow{O B 2} \text { c. } \vec{P}_{B}(j)-\vec{P}(j) \mid \\
& \overrightarrow{\text { Enclrcling }}_{O}=\mid \overrightarrow{O B 3} \text { c. } \vec{P}_{O}(j)-\vec{P}(j) \mid
\end{aligned}
$$

The hunting phase is followed by the attacking phase, pack attacks the prey. According to the position of the A.B and $\mathrm{O}$ the position of the other wolves are upgraded. This enables to attain the reliability in the solution. This foraging behavior is applied to the parameter attuning in the Power system modulator.

Proposed Algorithm: The primary conditions like the agents to search, maximum number of iterations, the total count of the parameters that has to be tuned, and the MIN and MAX limits and the starting values for the $\mathrm{A}, \mathrm{B}$ and the $\mathrm{O}$ and the estimated load is initiated. Followed by the estimation of error as well as fitness. The simulation file is executed to estimate the" Integral time square error over a period" with the basic values of the A, B, D in order to find out the fitness. Then update the A, B and D based on the score of the 
Journal of Electrical Engineering and Automation (EEA) (2020)

Vol.02/ No. 02

Pages: 102-111

https://www.irojournals.com/iroeea

DOI: https://doi.org/10.36548/jeea.2020.2.006

A, B and D, upgrade the positions of the other searching components and the $\mathrm{O}$. The distance of the every search component is upgraded using the equation (3)

$$
\left\{\begin{array}{l}
\text { Depth }(A)=\text { Fit1 postion }(A)-\text { optimal }(A) \\
\text { Depth }(B)=\text { Fit1 postion }(B)-\text { optimal }(B) \\
\text { Depth }(D)=\text { Fit1 postion }(D)-\text { optimal }(D) \\
\text { Depth }(O)=\text { Fit1 postion }(O)-\text { optimal }(O)
\end{array}\right.
$$

Where Fit $1=2 X$ random constant, depth is the distance and the optimal is best location. The parameters to be attuned are upgraded according to the locations of the search components, update the parameters to be estimated. Determine the miscalculations and the fitness by estimating the "Integral time square error" stop the process once the repetitions are completed and finally the optimal outcomes are displayed. The flow chart in figure. 1 describes the phase in the process of wolves foraging behavior to estimate the best parameters values.

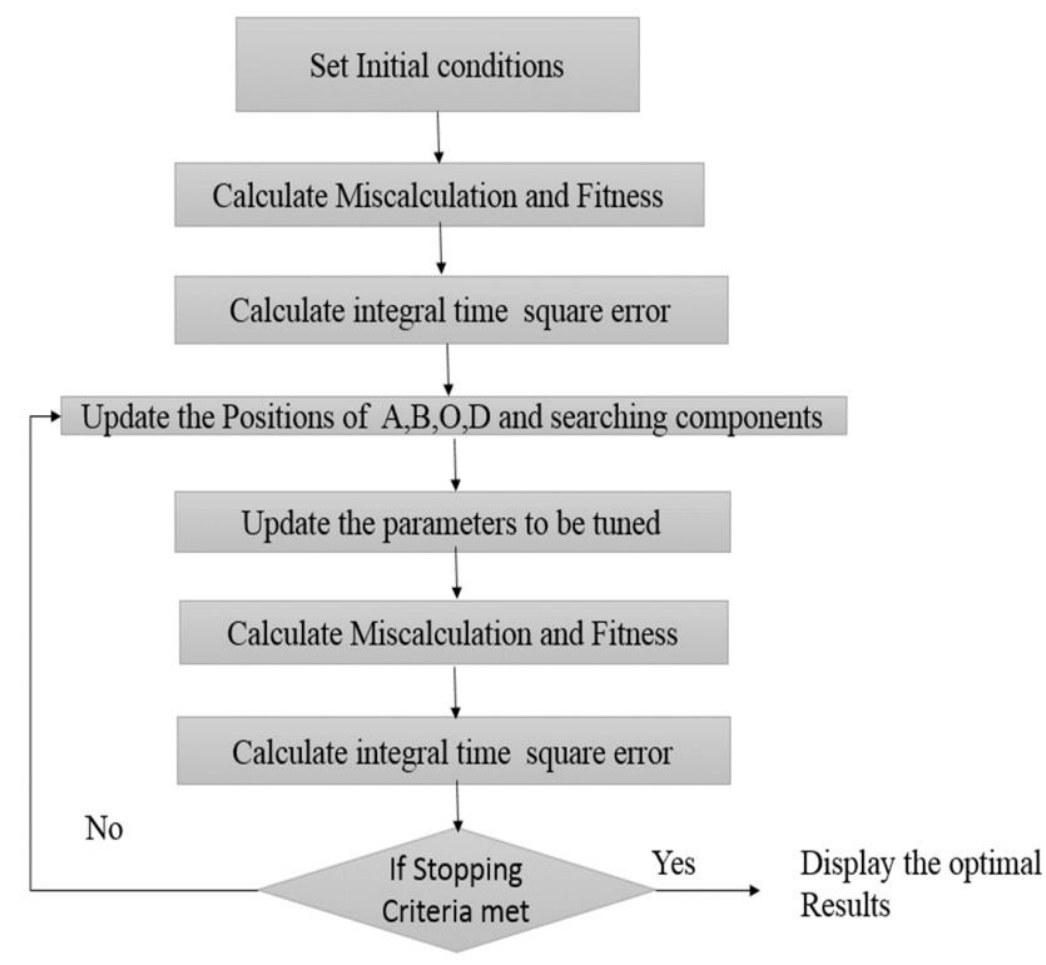

Figure.1 Proposed Flow Process of Wolves Foraging Behavior for Parameter Attuning In PSM 
Journal of Electrical Engineering and Automation (EEA) (2020)

Vol.02/ No. 02

Pages: 102-111

https://www.irojournals.com/iroeea

DOI: https://doi.org/10.36548/jeea.2020.2.006

\section{Performance Evaluation}

The GWO based parameter attuned PSM is evaluated on two test cases with multi machine system encompassed with two machineries and four buses. The figure. 2 depicts the 3-phase errors developed at the 1's.

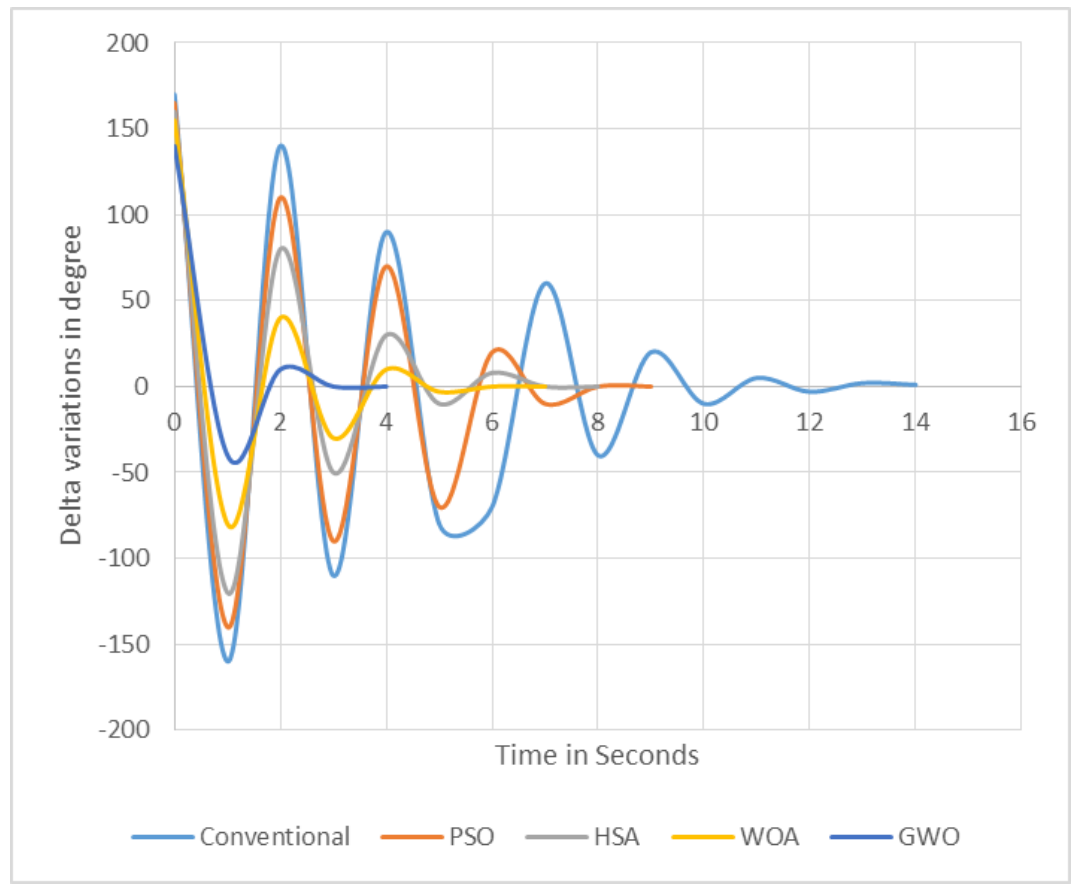

Figure.2 Delta Variations of PSM for Machine 2 W.R.T to Machine 1

The $\mathrm{D}$ aberrations of the device 2 W.R.T device 1 is depicted in figure 1 and the outcomes prove that the traditional methods shows 170 degree peak and settling time of more than 15 seconds. The other methods such as the WOA [7] HSA [3] and PSO [2] shows 155, 160, 165 degree peak respectively with the settling time of $6,7,8$ seconds respectively. But the proposed GWO based parameter attuned PSM shows 140 degree peak with 3 seconds settling period. The figure. 3 present the settling period of all the algorithms. 
Journal of Electrical Engineering and Automation (EEA) (2020)

Vol.02/ No. 02

Pages: 102-111

https://www.irojournals.com/iroeea

DOI: https://doi.org/10.36548/jeea.2020.2.006

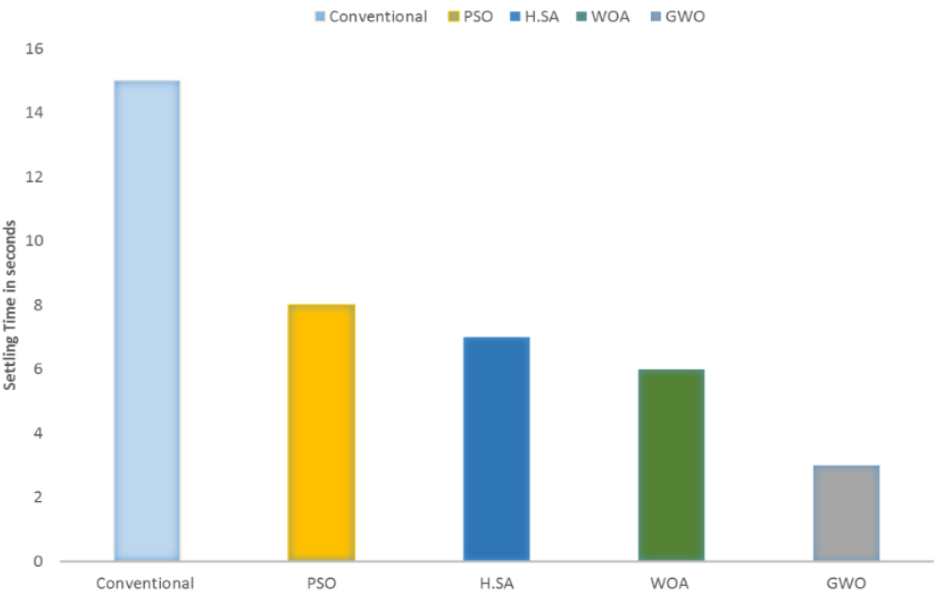

Figure.3 Settling period of Machine 2 W.R.T to Machine 1

The figure .4 depicts the speed variations of machine 2 with the PSM based on the conventional and the other algorithms like PSO, HSA and the WOA with respect to the machine 2 the outcome observed shows that the speed of the GWO based PSM measure in the radians/second is better compared to the speed variation of the other algorithms.

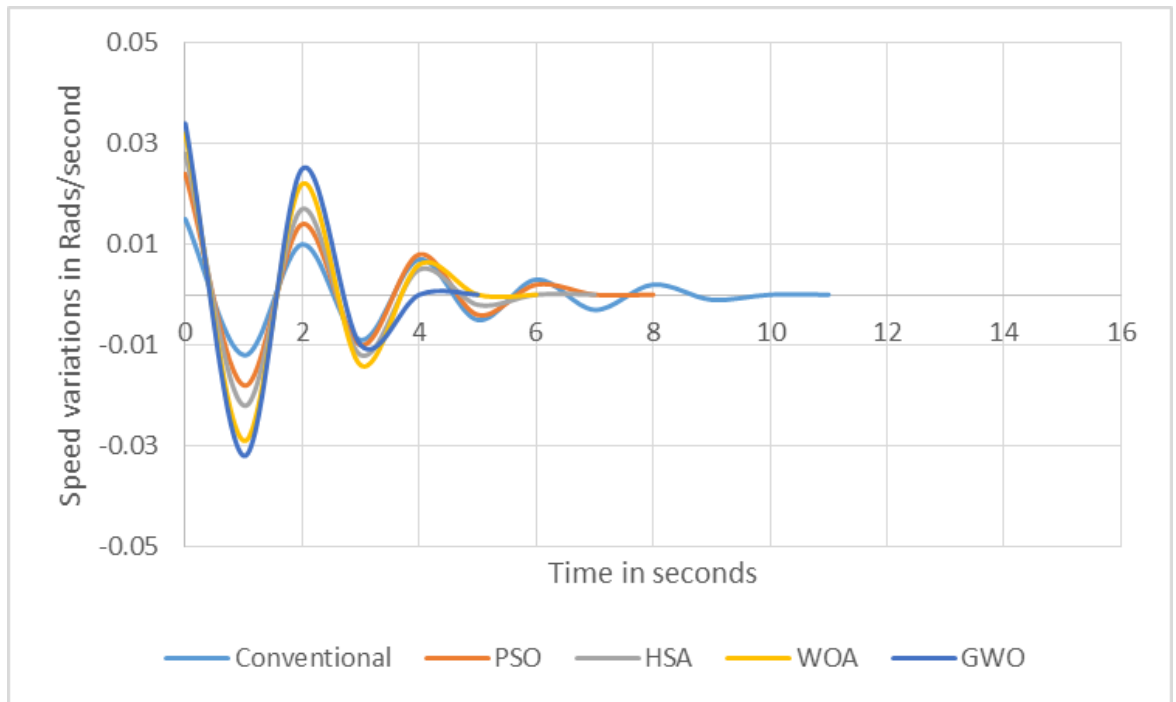

Figure.4 Speed Variations 
Vol.02/ No. 02

Pages: 102-111

https://www.irojournals.com/iroeea

DOI: https://doi.org/10.36548/jeea.2020.2.006

The speed variation with the PSO is measured as the .024, the HSA is measured as .028, and WOA is measured as .032 and the GWO based PSM is measured as 0.034 with respect to the machine 1 for machine 2 .

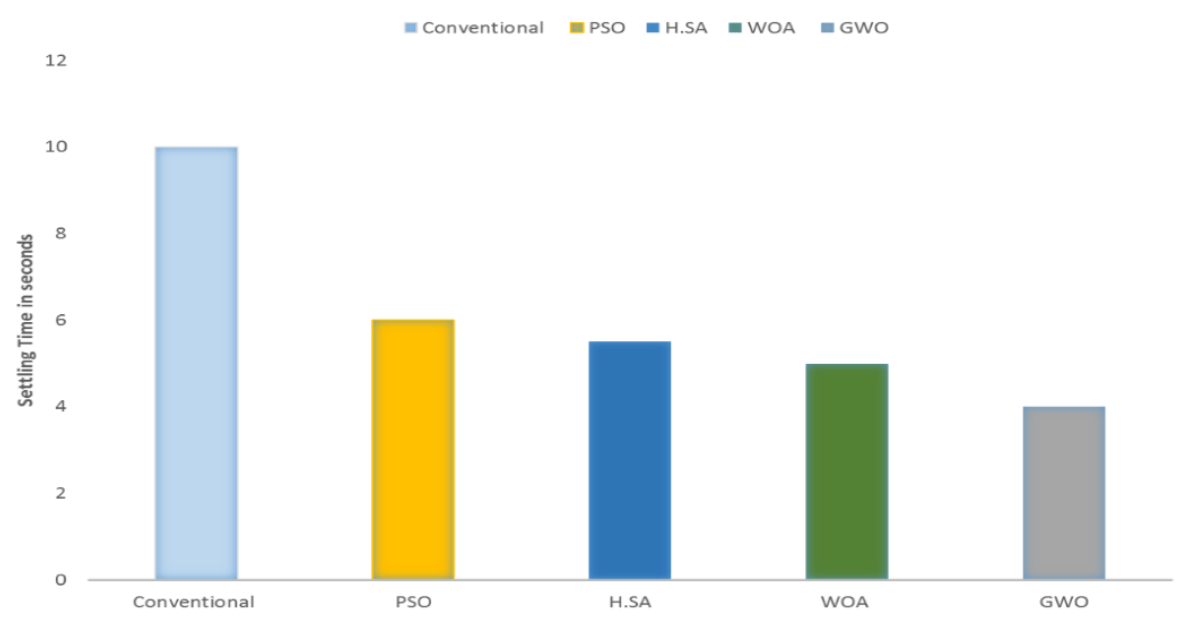

Figure.5 Settling Period

The settling time of the algorithms PSO, HSA, WOA and GWO and the conventional method are depicted in figure. 5 for the speed variations measured with respect to the machine 1 for the machine 2 . The settling time of PSO is observed as 6 seconds and the HSA is observed as 5.5 seconds and the WOA is 5 seconds and the GWO based PSM is 4 seconds.

\section{Conclusion}

The innovative procedure to attune the parameters of the power system modulator using the foraging behavior of the grey wolves in put forth in the paper. Based on the outcomes observed it is concluded that the devised way of attuning using the grey wolves hunting behavior, minimizes the overshoot of the peaks by maximum of 9.4 percentage and minimum of 2.38 percentage when compared to the traditional and the other algorithms. The settling period is also minimized effectively with the help of the proposed method based on the hunting activities of the grey wolves. The objective issue in stabilizing the operations and minimizing the fluctuations are perfectly managed using the proposed method. In future the paper is to proceed with further minimization of the peak overshoot and the settling period by hybridizing two algorithms that are bio-inspired with the scope of enhancing the stability and reducing fluctuations to make the operations more stable. 
Journal of Electrical Engineering and Automation (EEA) (2020)

Vol.02/ No. 02

Pages: $102-111$

https://www.irojournals.com/iroeea

DOI: https://doi.org/10.36548/jeea.2020.2.006

\section{References}

[1] http://www.meppi.com/Products/GeneratorExcitationProducts/Static\%20Excitation\%20Syste $\mathrm{m} /$ Power\%20System\%20Stabilizer.pdf

[2] El-Zonkoly, A. M. "Optimal tuning of power systems stabilizers and AVR gains using particle swarm optimization." Expert Systems with Applications 31, no. 3 (2006): 551-557.

[3] Naresh, G., M. Ramalinga Raju, and S. V. L. Narasimham. "Coordinated design of power system stabilizers and TCSC employing improved harmony search algorithm." Swarm and Evolutionary Computation 27 (2016): 169-179

[4] Ali, E. S., and S. M. Abd Elazim. "Power System Stabilizers Design Using Grasshopper Optimization Algorithm." International Journal of Power Systems 4 (2019).

[5] Sabo, Aliyu, Noor Izzri Abdul Wahab, Mohammad Lutfi Othman, Mai Zurwatul Ahlam Mohd Jaffar, and Hamzeh Beiranvand. "Farmland Fertility Optimization for Designing of Interconnected Multi-machine Power System Stabilizer." Applications of Modelling and Simulation 4 (2020): 183-201.

[6] Ekinci, Serdar, and Baran Hekimoglu. "Parameter optimization of power system stabilizer via salp swarm algorithm." In 2018 5th International Conference on Electrical and Electronic Engineering (ICEEE), pp. 143-147. IEEE, 2018.

[7] Dasu, Butti, Mangipudi Sivakumar, and Rayapudi Srinivasarao. "Interconnected multimachine power system stabilizer design using whale optimization algorithm." Protection and Control of Modern Power Systems 4, no. 1 (2019): 2.

[8] Gurrala, Gurunath, and Indraneel Sen. "Power system stabilizers design for interconnected power systems." IEEE Transactions on Power Systems 25, no. 2 (2010): 1042-1051.

[9] Lefebvre, S. "Tuning of stabilizers in multimachine power systems." IEEE Transactions on Power Apparatus and Systems 2 (1983): 290-299.

[10] Priyamvada, Indla Rajitha Sai, and Sarasij Das. "Robust Control based Energy Storage Stabilizers for Power Systems with Large Scale Renewables." In 2018 20th National Power Systems Conference (NPSC), pp. 1-6. IEEE, 2018.

[11] Nikolaev, A. A., A. S. Danilenko, and I. A. Lozhkin. "Study of Different Types of Power Systems Stabilizers used in Synchronous Generators Automatic Excitation Control Systems." Russian Internet Journal of Electrical Engineering 5, no. 1 (2018): 3-10.

[12] Chow, Joe H., and Juan J. Sanchez-Gasca. "Power System Stabilizers." (2020): 265-294.

[13] Raj, Jennifer S. "A Comprehensive Survey On The Computational Intelligence Techniques And Its Applications." Journal of ISMAC 1, no. 03 (2019): 147-159.

[14] Manikandan, R., and S. Smys. "Controller based performance measures of speed control of electrical motor for industrial applications." In 2017 International Conference on Inventive Systems and Control (ICISC), pp. 1-5. IEEE, 2017. 
Journal of Electrical Engineering and Automation (EEA) (2020)

Vol.02/ No. 02

Pages: 102-111

https://www.irojournals.com/iroeea

DOI: https://doi.org/10.36548/jeea.2020.2.006

[15] Faris, Hossam, Ibrahim Aljarah, Mohammed Azmi Al-Betar, and Seyedali Mirjalili. "Grey wolf optimizer: a review of recent variants and applications." Neural computing and applications 30, no. 2 (2018): 413-435.

\section{Authors Biography}

Dr. A. Dinesh Kumar, is currently working as professor in the department of Computer Science and Engineering, at Koneru Lakshmaiah Education Foundation, in Andra Pradesh, India. His major area of research are Digital Electronics, Robust Control, Industrial Automation, Adaptive Control, Process Control and Instrumentation and Power Systems. His current work carried out in the paper minimizes the fluctuation problems in the power systems by properly attuning of the parameters of power system modulator. 